\title{
Passivity-Based Full-Body Force Control for Humanoids and Application to Dynamic Balancing and Locomotion
}

\author{
Sang-Ho Hyon ${ }^{\dagger, \ddagger}$ \\ $\dagger$ Computational Brain Project, ICORP, JST \\ $\ddagger$ ATR, Computational Neuroscience Laboratories \\ Hikaridai 2-2-2, Souraku-gun, Kyoto 619-0288, Japan \\ E-mail:sangho@atr.jp
}

\author{
Gordon Cheng ${ }^{\dagger, \ddagger}$ \\ $\dagger$ Computational Brain Project, ICORP, JST \\ $\ddagger$ ATR, Computational Neuroscience Laboratories \\ Hikaridai 2-2-2, Souraku-gun, Kyoto 619-0288, Japan \\ E-mail: gordon@atr.jp
}

\begin{abstract}
This paper proposes a passivity-based hierarchical full-body motion controller for force-controllable multi-DOF humanoid robots. The task-space forces are treated in a uniform manner for a variety of position/force tracking and force/moment compensation. The contact force closure is optimally solved and transformed directly into the joint torques in real-time without any joint trajectory planning. With this framework, we introduce gravity compensation at the lowest layer of the controller that makes the closed-loop system passive with respect to additional inputs as well as external forces. Furthermore, we propose two upper-layers: one layer controls the ground reaction forces, which enables the robot keep the dynamic balance. The other layer is the another passification control, which constructs an invariant manifold that prevents the robot from falling during walking. Four realistic dynamic simulations: balanced squatting, reaching, externally driven, or speed-controlled walking with disturbances demonstrate the effectiveness of the proposed methods.
\end{abstract}

Index Terms-Humanoid robots, Full-body motion control, Balance, Locomotion, Passivity, Gravity compensation.

\section{INTRODUCTION}

\section{A. Background}

Full-body motion control is assigning DOFs (degrees of freedom) appropriately for given motion tasks. It originate from the control of redundant manipulator, where tracking performance for multiple objects, joint limitation, singular configuration or self-collision avoidance have been the main problems to be solved. For legged robots, two more essential control objectives were added; balancing and mobility. ZMP (Zero-Moment-Point [1]) or equivalently $\mathrm{CoP}$ (center of pressure) has been playing a central role for balancing [2][3][4].

The building and realization of multi-DOF humanoid robots are becoming familiar to us; more sophisticated motion control task by multi-DOF of hyper-DOF system is a growing interest within the humanoid research community. For such a high dimensional system the reduction of dimensionality is the central issue from the viewpoint of real-time motion control. Since the center of mass (CoM) of a multi-DOF system represents the integrated translational motion behavior of the whole system, it is clearly a promising controlled variable of the moving objects in Cartesian space.

For mobile robot such as (biped) humanoid robots, the location of CoM serves not only as a direct target to be reached but also as an important index for balancing. Controlling CoM

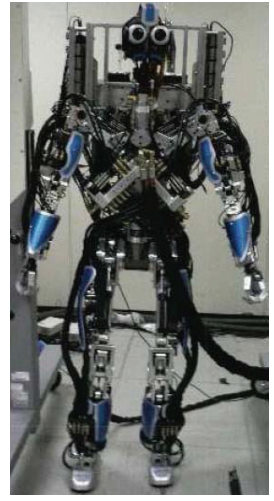

(a) Full-body force controllable humanoid robot built by SARCOS Inc.

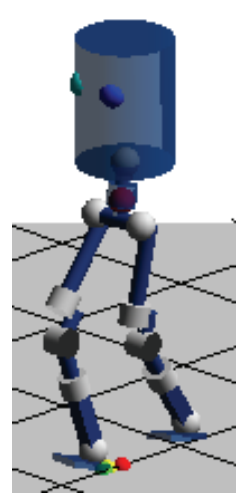
model I (7DOF for legs and $3 \mathrm{DOF}$ for torso)

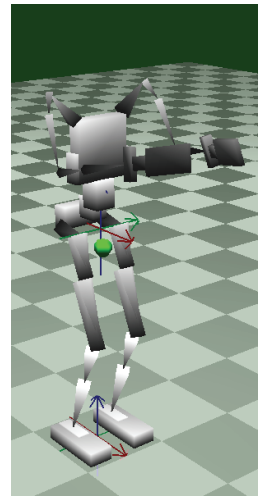

(c) Simulation model II (7DOF for legs and arms and $3 \mathrm{DOF}$ for torso)
Fig. 1. Humanoid robot models considered in this paper

via ZMP modulation was proposed by Honda and successfully applied to their real humanoid robots [3]. The idea was further analyzed in [5][6] and extended to whole-body motion control in [7][8] where position-controllable humanoids are assumed. The redundancy problem was solved by weighted pseudoinverse of Jacobian from ZMP to CoM. Direct acceleration control of CoM for a multi-DOF humanoid robot is proposed in [9][10] where torque-controllable robots are assumed and resolved acceleration controller (with a perfect model matching assumption) was employed. Recently, [11] proposed a new task space controller for force-controllable humanoids, where the task is projected onto the constraint nulll-space and the recursive algorithm via multi-level prioritization was developed, but how to deal with dynamic balancing or walking have not been presented.

\section{B. Scope and contribution}

This paper proposes simple and practical full-body force controllers for a force-controllable humanoid robot (Fig. 1(a)) and presents some simulation results on real-time balancing and walking. The details of the robotic hardware will be 
presented in [12], and some initial walking results can be found in [13]. The controllers developed in this paper are general and do not assume any specific models, but only assume the joint torques are controllable.

First we review our hierarchical motion control strategy based on passivity [14][15] in Section II. We will explain how the different controllers presented in this paper are effectively combined in a hierarchical manner. In Section III, we propose a novel force control framework. Therein, the task-space forces are treated in a uniform manner which are used for various position/orientation control as well as force interaction, and the contact forces are optimally distributed in real-time. Different from the recently-proposed methods [11], we do not calculate all the nonlinear terms and cancel them, while maintaining good contact force tracking performance.

With this force control framework, we will present some motion examples on balancing and walking in Section V. In the balancing examples, CoM tracking control, orientation control and reaching control are demonstrated. In the walking examples, the externally-driven walking and speed-controlled walking with disturbances are shown. In particular, Symmetric Walking Control (SWC) proposed in [15] is generalized to multi-DOF humanoid robots in Section IV.

Under the assumption of force-controllability within the interested bandwidth, our controller has the following benefits:

- No need for pre-calculation of joint trajectories;

- A few modeling parameters (no inertia matrix);

- Easy task addition;

- Applicable to arbitrary contact points;

- No need to assign the weights, as opposed to positionbased full-body controllers;

- Uniform treatment of position/orientation control;

- External-drivability (force interaction).

The effectiveness of the proposed controller and the further extensions are discussed in Section VI. The experimental results will be presented in the very near future [16].

\section{Hierarchical motion CONTROL StRategy based ON PASSIVITY}

The purpose of the control design here is to generate robust motions not only in real-time but also autonomously without relying on pre-planned reference trajectories, but with achieving simple task objectives, such as walking speed or reaching point. For this purpose, we propose a motion control strategy based on passivity of mechanical systems [14][15]. The strategy comprises of three steps, as shown in Fig. 2:

(S1) First, we disregard dissipativity of the system to extract purely Hamiltonian (conservative) dynamics. Then, apply invariance control that render the closed-loop system lossless and restricts the hybrid flows into some invariant sets to obtain a family of Lyapnov stable periodic orbits or chaotic orbits.

(S2) Retrieve (and inject as necessary) dissipativity and apply a tracking controller to obtain asymptotically stable target states or periodic orbits passing through the desired target states.

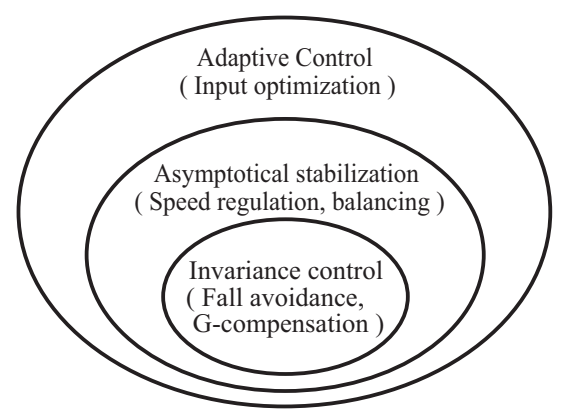

Fig. 2. Concept of the hierarchical motion control strategy composing of: invariance control, asymptotical stabilization and adaptive control.

(S3) Apply adaptive or learning schemes to minimize control efforts.

The resultant controller has a hierarchical structure in which the existence and the boundedness of the solutions must be ensured at the lowest layer with a high priority; more advanced control goals should be achieved at the higher layers. In this strategy, the invariance control (S1) plays the most important role. As shown in [14], the invariance control can be regarded as an extension of the feedback passification (see the following sentences) to a class of hybrid systems.

The concept of passivity has played an important role in the stabilization of nonlinear control systems [17]. The passivity is closely related to the stability since the closedloop system possess Lyapnov stable zero dynamics. When the equilibrium point of original Hamiltonian is not related to control task, however, some feedback controller must be applied to produce a new (shaped) Hamiltonian. How can a nonlinear system be rendered passive via feedback is called a (feedback) passification, or feedback equivalence to a passive systems. The gravity compensation control briefly described in Section III-C achieves a passification for the humanoid robots (Fig. 2) as shown in [18].

It is very important to note that a merely passified system, however, is not sufficient to obtain invariant sets when there are some inadmissible regions where the motion is impossible (due to the unilateral constraints, friction, and so forth). Therefore, the controller must bound the solution inside the admissible region as well as passify the system. Rendering the controlled system lossless with respect to some supply rate and bounding the solution into some admissible region is called invariance control in [14].

The SWC presented in Section IV is one of the invariance control that makes the configuration bounded by imposing a control constraint and switching logic, resulting in another passified system. It has been theoretically proven in [15] that, as long as the robot keeps contact with the ground, it does not fall. ${ }^{1}$ The invariance control attributes flexibility in subtasks (provided additional active DOFs are available) in the upper layer (S2). For example, a speed-controllable and

\footnotetext{
${ }^{1}$ In [15] the authors claimed the global stability, but it was actually the semi-
} global or regional stability determined by a given stride (design parameter). 
asymptotically orbitally stable walking can be achieved by simply combining the SWC with a simple CoM feedback law as demonstrated in Section V-C.

\section{NOVEL FORCE CONTROL FRAMEWORK}

This section presents a novel force control framework for humanoids with multiple contact points and the force interaction points. The force control frameworks is general; it can be applied to arbitrary contact points. Our method is different from recently published paper [19] in that we consider dynamic balance and optimal load distribution. Section III-A describes general formulation of controlling CoM via gross applied force (defined therein). Section III-B explains how to distribute the gross applied force to multiple contact points through ZMP. Section III-C shows how to transform the desired contact forces to joint torques. Section III-D shows how to deal with controlling the swinging legs or hands for reaching tasks or force interaction tasks. Section III-E briefly describes how to generate rotational moments and achieve desired orientations of the base, hands, etc, and how to compensate yaw moment.

\section{A. General formulation of CoM control}

Consider a multi-DOF humanoid robot as shown in Fig. 3 . Let $q \in \mathcal{S}^{n}$ be the joint angles where $n$ is the number of the joints. The important control objective of multi-DOF humanoid robots is controlling CoM since it represents the integrated translational motion behavior of the whole system. Let $r_{C}=\left[x_{C}, y_{C}, z_{C}\right]^{T} \in \mathcal{R}^{3}$ be the position vector of CoM in the world coordinate frame $\Sigma_{W}$.

Consider the exact nonlinear dynamics of humanoids with a single point constraint expressed by

$$
\begin{aligned}
\hat{I}(\hat{q}) \ddot{\hat{q}}+\hat{C}(\hat{q}, \dot{\hat{q}})+\hat{G}(\hat{q}) & =u+E(\hat{q})^{T} \lambda \\
E(q) \dot{\hat{q}} & =0
\end{aligned}
$$

with $\hat{q}=[r, q]^{T} \in \mathcal{R}^{3+n}$ the generalized coordinates including the translational coordinates $r \in \mathcal{R}^{3}$ in $\Sigma_{W}, \hat{I}(\hat{q})$ the inertia matrix, $\hat{C}(\hat{q}, \dot{\hat{q}})$ the centrifugal and Coriolis term, $\hat{G}(\hat{q})$ the gravity term, $u=[0, \tau]^{T} \in \mathcal{R}^{3+n}$ the generalized forces, $E(q)$ the constraint Jacobian associated with the point

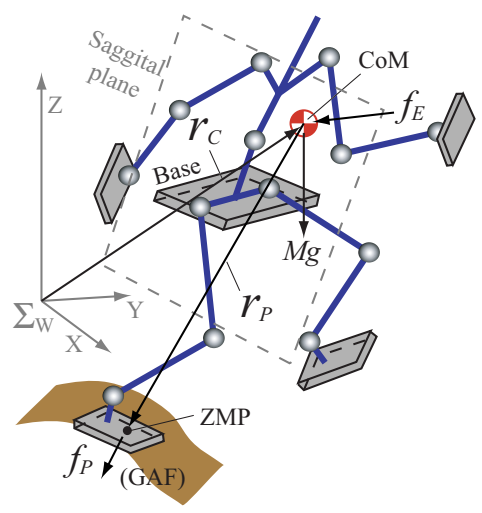

Fig. 3. CoM, ZMP, and GAF

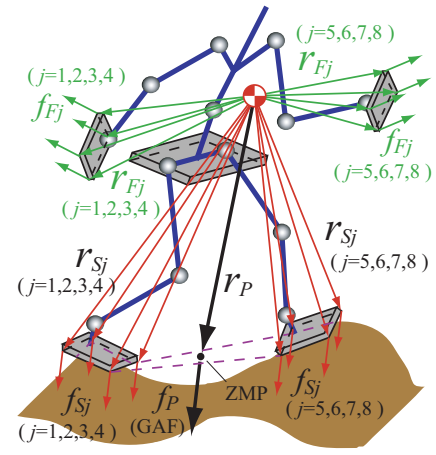

(a) Unilateral contact (support)

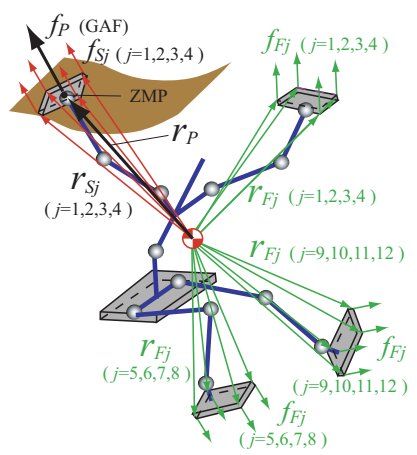

(b) Bilateral contact (hung)
Fig. 4. Definition of GAF, contact points and forces: Each contact point $r_{S j}$ is assigned with the contact force $f_{S j}$, and each floating point $r_{F j}$ is assigned with the interaction force $f_{F j}$. The contact forces $f_{S j}$ are determined by a desired GAF $f_{P}$ and $f_{F j}$. Note that ZMP $r_{P}$ always lies within the supporting convex hull composed of $r_{S j}$.

constraint, and $\lambda \in \mathcal{R}^{3}$ the constraint force. See [20] for its derivation.

The above equation may becomes very complicated according to the selection of the translational coordinates and the constraint. Therefore, the relationship between ZMP and CoM is very important. Let $r_{P}=\left[x_{P}, y_{P}, z_{P}\right]^{T} \in \mathcal{R}^{3}$ be the position vector from CoM to ZMP (Fig. 3). Note that ZMP is not fixed, but moving on the ground surface.

Now, let us choose ZMP as the single contact point and $\mathrm{CoM}$ as the translational coordinate respectively, so that the generalized coordinates are $\hat{q}=\left[r_{C}, q\right]^{T} \in \mathcal{R}^{3+n}$. Then, (1) is transformed to the decoupled dynamics:

$$
\begin{aligned}
{\left[\begin{array}{c|c}
M & 0 \\
\hline 0 & I(q)
\end{array}\right]\left[\begin{array}{c}
\ddot{r_{C}} \\
\hline \ddot{q}
\end{array}\right] } & =\underbrace{\left[\frac{0}{C(q, \dot{q})}\right]}_{\hat{C}}+\underbrace{\left[\frac{M g}{0}\right]}_{\hat{G}} \\
& +u+E(q)^{T} f_{P},
\end{aligned}
$$

where $M=\operatorname{diag}[m, m, m]$ with $m$ the total mass, $g=$ $[0,0,-9.81]^{T}$, and

$$
E(q)=\left[-i d \mid \frac{\partial r_{P}}{\partial q}\right] .
$$

In (3) we have introduced a gross applied force (GAF) $f_{P}=$ $\left[f_{x P}, f_{y P}, f_{z P}\right]^{T}$, defined as $f_{P}:=-\lambda$, the force the robot applies to the environment. Note that the upper part of (3) shows a simple linear dynamics

$$
M \ddot{r}_{C}=M g-f_{P}
$$

and hence we can control $\ddot{r}_{C}$ by $f_{P}$.

From (1) and (2) $f_{P}$ can be written as

$$
f_{P}=\left(E I^{-1} E^{T}\right)^{-1}\left\{\gamma+E I^{-1}(u-\hat{C}-\hat{G})\right\}
$$

with $\gamma(q, \dot{q})=\frac{\partial}{\partial q}(E \dot{q}) \dot{q}$. Therefore, for some desired $\bar{f}_{P}$ we can calculate the corresponding joint torques by inverting (6). See [10] for more details on this type of GAF control and 
its experimental validation in dynamic whole-body control of under-actuated systems.

\section{B. Optimal contact force distribution via ZMP}

The GAF derived in (6) is not enough to make humanoid robots interact with the environment at multiple contact points. The subject of this section is how to distribute GAF to multiple contact points. To treat the force control in task space in an uniform manner, we consider translational positions and forces of the interested force application points. Fig. 4 shows such force application points, as well as GAF. Note that all the position vectors run from CoM. Assume we are interested in total $\alpha$-contact points defined by $r_{S}=$ $\left[r_{S 1}, r_{S 1}, \cdots, r_{S \alpha}\right]^{T} \in \mathcal{R}^{3 \alpha}$ and the associated contact forces $f_{S}=\left[f_{S 1}, f_{S 1}, \cdots, f_{S \alpha}\right]^{T} \in \mathcal{R}^{3 \alpha}$. Of course, $r_{P}$ must lie within the supporting convex hull composed of $r_{S j}$. We are also interested in total $\beta$-floating points defined by $r_{F}=\left[r_{F 1}, r_{F 1}, \cdots, r_{F \beta}\right]^{T} \in \mathcal{R}^{3 \beta}$ and the associated applied forces $f_{F}=\left[f_{F 1}, f_{F 1}, \cdots, f_{F \beta}\right]^{T} \in \mathcal{R}^{3 \beta}$ For example, $\alpha=8$ and $\beta=8$ in Fig. 4(a), but $\alpha=4$ and $\beta=12$ in Fig. 4(b).

The relationship among GAF, ZMP and the contact positions/forces can be written as

$$
\begin{gathered}
f_{P}=\sum_{j=1}^{\alpha} f_{S j}, \\
x_{P}=\frac{\sum_{j=1}^{\alpha} x_{S j} f_{z S j}}{\sum_{j=1}^{\alpha} f_{z S j}} \quad, \quad y_{P}=\frac{\sum_{j=1}^{\alpha} y_{S j} f_{z S j}}{\sum_{j=1}^{\alpha} f_{z S j}},
\end{gathered}
$$

or, in the simple notation

$$
f_{z P}\left[\begin{array}{c}
x_{P} \\
y_{P} \\
1
\end{array}\right]=\underbrace{\left[\begin{array}{cccc}
x_{S 1} & x_{S 2} & \cdots & x_{S \alpha} \\
y_{S 1} & y_{S 2} & \cdots & y_{S \alpha} \\
1 & 1 & \cdots & 1
\end{array}\right]}_{A_{z} \in \mathcal{R}^{3 \times \alpha}}\left[\begin{array}{c}
f_{z S 1} \\
f_{z S 2} \\
\cdots \\
f_{z S \alpha}
\end{array}\right],
$$

where $A_{z}$ represents a contact force distribution matrix. For some desired normal GAF $\bar{f}_{z P}$ and ZMP $\bar{r}_{P}$ we can calculate the corresponding desired contact forces ${ }^{2}$. Specifically, this paper proposes an optimal contact force distribution given by

$$
\left[\begin{array}{c}
\bar{f}_{z S 1} \\
\bar{f}_{z S 1} \\
\cdots \\
\bar{f}_{z S \alpha}
\end{array}\right]=A_{z}^{\# \bar{f}_{z P}}\left[\begin{array}{c}
\bar{x}_{P} \\
\bar{y}_{P} \\
1
\end{array}\right]
$$

with $A_{z}^{\#}=A_{z}^{T}\left(A_{z}^{T} A_{z}\right)^{-1}$, because it is optimal in the sense that it minimizes the norm of the total contact forces.

Similarly we can distribute desired horizontal GAFs, $\bar{f}_{x P}$ and $\bar{f}_{y P}$, to the corresponding desired horizontal contact forces $\bar{f}_{S x j}$ and $\bar{f}_{S y j}$ through $A_{x}$ and $A_{y}$ (omitted).

Consequently, a desired GAF is distributed to desired contact forces by a simple matrix operation

$$
\bar{f}_{S}=A^{\#} \bar{f}_{P}\left[\begin{array}{c}
\bar{x}_{P} \\
\bar{y}_{P} \\
1
\end{array}\right],
$$

${ }^{2}$ For ZMP-constrained balancer, the desired ZMP is determined by $\bar{f}_{P}$, then truncated by the supporting convex hull. where the contact force distribution matrix $A$ is obtained by permutating $A_{x}, A_{y}$ and $A_{z}$. Note that (5) only considers controlling translational motions by $f_{P}$. But the yaw moment compensation is also crucial. See Section III-E.

\section{Commanded joint torque with gravity compensation}

The desired contact forces (11) is converted to commanded joint torques simply by

$$
\tau=J_{S}(q)^{T} \bar{f}_{S}+D \dot{q}+\tau_{a}
$$

where $J_{S}(q) \in \mathcal{R}^{3 \alpha \times n}$ represents the contact Jacobian from CoM to supporting contact points (derivative of $r_{S}$ by $q$ ), and $D \in \mathcal{R}^{n \times n}$ is the joint-wise damping coefficient matrix to supress the internal motions [21]. How to chooe $D$ is discussed in [16] in the context of the "contact force control". ${ }^{3} \tau_{a}$ is additional joint torque used for joint-space tracking tasks with possibly higher priority, such as posture control (Section V-B).

This torque control works well only if the gravity term $M g$ in (6) is compensated because the first RHS term of (12) is based on static force relationship as explained in [16]. Therefore, we set the desired GAF as

$$
\bar{f}_{P}=f_{u}-M g,
$$

with some new force inputs $f_{u}$. The gravity compensation is one of the invariance control (S1) at the bottom layer, while $f_{u}$ is left for the upper layer control (S2) (see Section II). Details of the gravity compensation and its importance in contact force control is fully discussed in [16].

\section{Reaching or force-interaction tasks}

We can use the interaction forces $f_{F j}$ for controlling the position $r_{F j}$ of the swinging leg (or hands) by, for example, a simple PD feedback law

$$
\begin{array}{r}
\bar{f}_{F j}=-K_{P F}\left(r_{F j}-\bar{r}_{F j}\right)-K_{D F}\left(\dot{r}_{F j}-\dot{\bar{r}}_{F j}\right), \\
(j=1,2, \cdots, \beta)
\end{array}
$$

with some desired positions/velocities $\bar{r}_{F j}, \dot{\bar{r}}_{F j}$ and positive gain matrix $K_{P}, K_{D}>0$, or by some feed-forward control. The force-interaction is much easier (by simply setting $\bar{f}_{F j}$ as desired).

To achieve good tracking of a desired GAF $\bar{f}_{P}$ the interaction forces must be compensated. ${ }^{4}$ This can be done by simply subtracting the sum of the interaction forces from the desired GAF in advance:

$$
\bar{f}_{P} \stackrel{\text { substitute }}{\longleftarrow} \bar{f}_{P}-\sum_{j=1}^{\beta} \bar{f}_{F j} .
$$

Thus with this contact force framework the motion command for supporting leg joints or swinging leg joints can be handled in a uniform manner. The toe-off contact or the heel-off contact can be treated as well.

\footnotetext{
${ }^{3}$ For general description on this type of redundancy resolution in robotic manipulators, see [22], where the design of $D$ is discussed in the context of "position tracking control" of the end-effector.

${ }^{4}$ If task space feedback is employed and the motion is slow enough, the force/moment compensation is not necessary.
} 
Not only the position of the foot, but also the orientation can be easily controlled. We will omit it because of the page limit, but refer to the next section to see how the contact forces generate moment.

\section{E. Moment generation by contact forces}

The contact forces can be also used for moment compensation. For example, let $\tau_{z}$ be the applied yaw moment. It is related to the contact forces by

$$
\tau_{z}=\sum_{j=1}^{\alpha}\left[\begin{array}{l}
x_{S j} \\
y_{S j}
\end{array}\right] \times\left[\begin{array}{l}
f_{x S j} \\
f_{y S j}
\end{array}\right] \in \mathcal{R}^{1},
$$

or, in a simple notation

$$
\tau_{z}=\underbrace{\left[-y_{S 1}, x_{S 2}, \cdots,-y_{S \alpha}, x_{S \alpha}\right]}_{B \in \mathcal{R}^{1 \times 2 \alpha}}\left[\begin{array}{c}
f_{x S 1} \\
f_{y S 1} \\
\cdots \\
f_{y S \alpha} \\
f_{y S \alpha}
\end{array}\right]
$$

where $B$ represents a contact moment distribution matrix. For a desired yaw moment $\bar{\tau}_{z}$ we can calculate the corresponding contact forces by

$$
\left[\begin{array}{c}
\bar{f}_{x S 1} \\
\bar{f}_{y S 1} \\
\cdots \\
\bar{f}_{y S \alpha} \\
\bar{f}_{y S \alpha}
\end{array}\right]=B^{\#} \bar{\tau}_{z} .
$$

This paper proposes the following two desired moments. The first one is to control the base heading:

$$
\bar{\tau}_{z 1}=-K_{P Y}\left(e_{3}-\bar{e}_{3}\right)-K_{D Y} \dot{e}_{3}
$$

with some positive gain $K_{P}, K_{D}>0$, where $e_{3}$ is the yaw orientation of the base. The second one is to compensate the yaw moment due to the swinging leg's (or trunk's or hands') motion:

$$
\bar{\tau}_{z 2}=-\sum_{j=1}^{\beta}\left[\begin{array}{c}
\left(x_{F j}-x_{P}\right) \\
\left(y_{F j}-y_{P}\right)
\end{array}\right] \times\left[\begin{array}{c}
\bar{f}_{x F j} \\
\bar{f}_{y F j}
\end{array}\right] .
$$

The total target moment $\bar{\tau}_{z}=\bar{\tau}_{z 1}+\bar{\tau}_{z 2}$ is then substituted into (18) to modify the contact forces by adding them into the original contact forces, as in (15), that is,

$$
\bar{f}_{S} \stackrel{\text { substitute }}{\longleftarrow} \bar{f}_{S}+\sum_{j=1}^{\alpha} \bar{f}_{S j} \text {. }
$$

In the same way, the orientation control for swinging legs or hands can be easily implemented.

\section{FULL-BODY SYMMETRIC WALKING CONTROL}

This section generalizes SWC proposed in [15] to multiDOF humanoids. Fig. 5 summarizes the related coordinates. Note that the heading plane and the sagittal plane are not necessarily aligned. Let $r_{Q}=\left[x_{Q}, y_{Q}, z_{Q}\right]^{T} \in \mathcal{R}^{3}$ be the position vector from CoM to the center of the swinging foot.

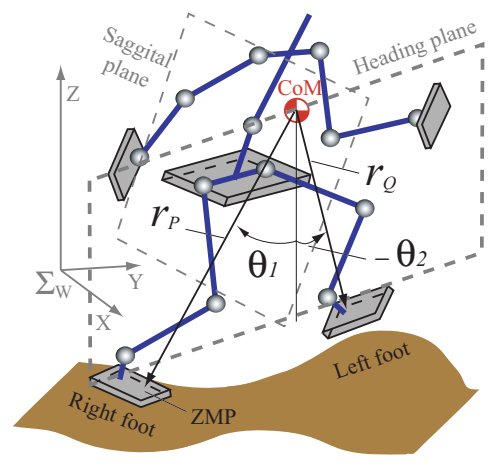

Fig. 5. Illustration of 3D symmetric walking: $r_{P}$ and $r_{Q}$ are the vector from $\mathrm{CoM}$ to ZMP and to the center of swinging foot respectively. $\theta_{1}$ and $\theta_{2}$ are the associated angle variables.

The controller is very simple; it only constraints the motion of the swinging leg by

$$
h(x)=\left[\begin{array}{c}
x_{P}+x_{Q} \\
y_{P}+y_{Q} \\
z_{P}-z_{Q}-\bar{z}_{Q}
\end{array}\right]=0 .
$$

The desired height of the swinging foot $\bar{z}_{Q}$ specifies a ground clearance; it is a function of the distance $d_{x y}:=$ $\sqrt{\left(x_{Q}-x_{P}\right)^{2}+\left(y_{Q}-y_{P}\right)^{2}}$. For a given stride $\bar{d}_{x y}$ (design parameter), $\bar{z}_{Q}$ must be zero when $\left|d_{x y}\right| \geq \bar{d}_{x y}$ to make the exchange of supporting leg. We are using a very simple linear function. Note that during walking the direction of the heading plane may changes, and the robot moves its foot to the direction of heading [15].

SWC is not limited to gaits of fixed CoM height. The only requirement is: the horizontal position of the swinging foot being symmetric to that of supporting foot. But, practically, maintaining the CoM height constant helps on preventing the knee from over-extended. Once the over-extension happens, the knee joint torque may become zero. It will be important to analyze the relationship between the design parameters (CoM height and the stride) to obtain optimal walking gaits.

The mathematical properties of SWC presented in [15] are met in the same way by considering the angle variables $\theta_{1}$ and $\theta_{2}$ in Fig. 5. That is, controlled symmetric orbits densely distributed in the whole interested phase space limited by the switching stride. See Section V-C for further properties.

\section{SimUlation}

\section{A. Simulation model}

The controllers developed are evaluated on two dynamic simulation models. The first model (Fig. 1(b)) has two 7-DOF legs and a 3-DOF HAT (head, arms and trunk). We assume the total mass be $m=75 \mathrm{~kg}$ ( $14.2 \mathrm{~kg}$ for each leg, $7.5 \mathrm{~kg}$ for base and $39.1 \mathrm{~kg}$ for HAT) and the hip height be $0.7 \mathrm{~m}$. The sole is a cubic solid of $0.1 \mathrm{~m}$ in width, $0.15 \mathrm{~m}$ in length and $0.01 \mathrm{~m}$ in depth. The simulator is built on SD/FAST, where the ground contact is modeled as unilateral virtual springs and dampers attached to the each corner of the sole (see Fig. 4(a)). 
The second model (Fig. 1(c)) has two 7-DOF legs, two 7DOF arms and a 3-DOF trunk. The simulator is also built on $\mathrm{SD} / \mathrm{FAST}$, but the ground contact is modeled in an advanced way [23].

For CoM tracking, a simple PD feedback is employed:

$$
f_{u}=-K_{P C}\left(r_{C}-\bar{r}_{C}\right)-K_{D C}\left(\dot{r}_{C}-\dot{\bar{r}}_{C}\right) .
$$

We use the following control parameters consistently in the all simulations (the gain matrices are diagonal whose elements have the same value otherwise specified)

- $\left(K_{P C}, K_{D C}\right)=(5000,500)$ for CoM height control, and $\left(K_{P C}, K_{D C}\right)=(100,50)$ for horizontal CoM control in (23),

- $\left(K_{P F}, K_{D F}\right)=(1000,10)$ for swinging leg tracking control (14),

- $\left(K_{P Y}, K_{D Y}\right)=(1000,10)$ for yaw moment compensation (19),

- $\left(K_{P A}, K_{D A}\right)=(1000,10)$ for torso attitude control $(24)$,

- $d_{\text {legs }}=2$ and $d_{\text {torso }}=8$ for joint-wise damping (12).

The tracking performance obviously depends on the gains. It is important to note, however, that tracking tasks are not necessarily achieved by these kinds of very simple feedback controllers only. Feed-forward compensator definitely improves the performance. An important thing is considering the required forces into the contact forces as (15) or (21).

\section{B. Dynamic balancing}

Here we will show some ZMP-constrained balancing simulations. The origin of the robot is set to the center of the feet. In the simulation model Fig. 1(b), ZMP, and hence $\bar{r}_{P}$, can travel from $-0.05 \mathrm{~m}$ to $+0.1 \mathrm{~m}$ in $X$-direction when the both feet are aligned. GAF $f_{u}$ is then truncated accordingly. First we show a balanced squatting with the torso orientation tracking. We use $\bar{x}_{C}=\bar{y}_{C}=0$ and give some reference trajectory for $\bar{z}_{C}$ in (23). The orientation control for torso can be done either by assigning the interaction forces as in Section III-D or simply by a local feedback

$$
\tau_{A}=-K_{P A}(\phi-\bar{\phi})-K_{D A}(\dot{\phi}-\dot{\bar{\phi}})=: \tau_{a},
$$

which is added to RHS of (12). In the same way we can add the joint space control as required. But note that this addition is valid only if the total contact Jacobian $J_{C}$ in (12) has full rank. Otherwise, we cannot achieve the tracking.

Fig. 6 and Fig. 7 show the simulation result. The desired $\mathrm{CoM}$ height and the torso orientation are simply given by sinusoidal trajectories as shown in Fig. 7. No feed-forward compensation discussed in Section III-E is applied. To prevent the robot from take-off, we made a lower bound for $\bar{f}_{z u}$. Fig. 8 shows a squatting on the full-body dynamic simulator [23]. Note that the contact forces are distributed to whole-body including the arms (the arm joints are moving).

Fig. 9 shows another balancing task; continuous reaching task. The CoM height $z_{C}$ is not controlled but largely damped with the same derivative gain, $K_{D C}$. The position of the swinging leg, $r_{F}$, is given as a sinusoidal function as shown in

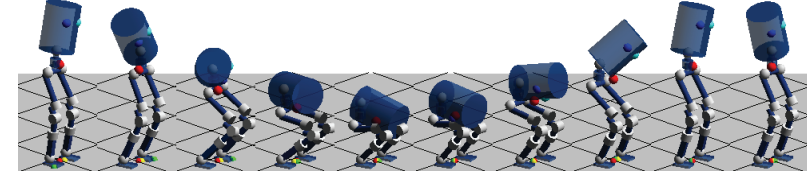

Fig. 6. Animation of a balanced squatting with torso swinging for 1-3 sec: two red markers shows CoM and $\mathrm{gCoM}$, while yellow and green ones indicate the desired ZMP and actual ZMP.
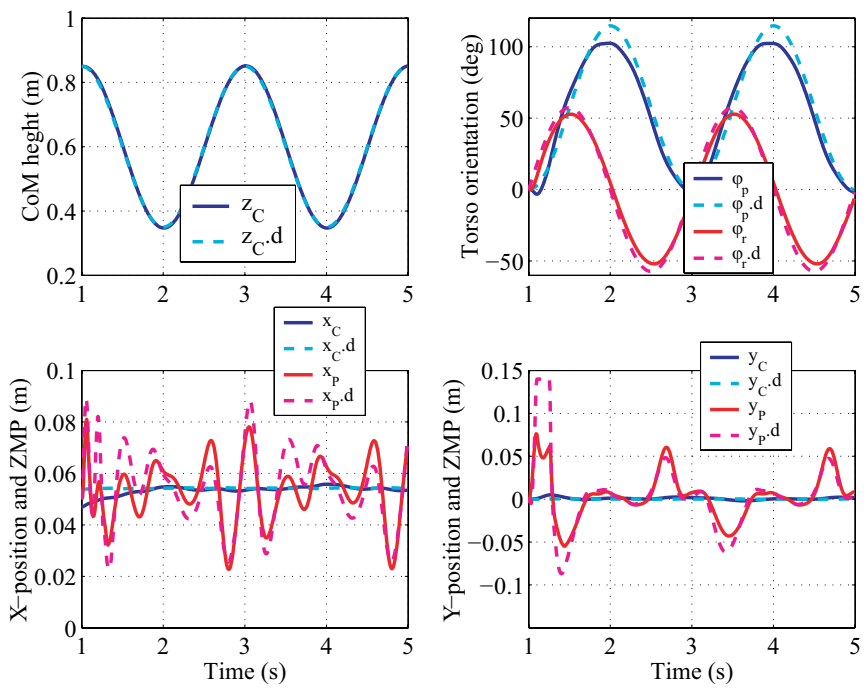

Fig. 7. Time evolution the balanced squatting in Fig. 6: CoM $\left(x_{C}, y_{C}, z_{C}\right)$ and ZMP $\left(x_{P}, y_{P}\right)$ are shown with their desired values (indicated by ".d").

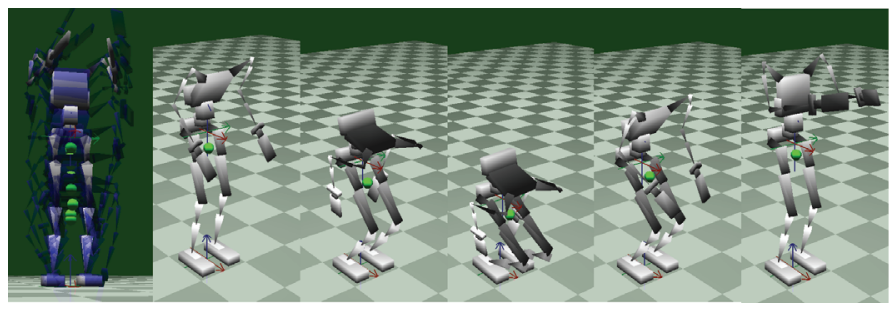

Fig. 8. Squatting on a full-body humanoid simulator [23]

Fig. 10. During the motion, the target equilibrium point, that is, the center of the supporting foot, is slightly moving due to the slip. Nevertheless, the horizontal CoM position $x_{C}, y_{C}$ is stabilized to the new equilibrium (the center of the sole).

\section{Dynamic walking}

Fig. 11 shows a simulation result of SWC, where the robot suffers from a large amount of external forces during walking. In this simulation the balancing controller is de-activated, that is, $\bar{f}_{x u}=\bar{f}_{y u}=0$ is enforced in (23), to purely demonstrate the performance of the invariance controller, while the CoM height is controlled to $0.8 \mathrm{~m}$ (see Section IV for the reason). The switching stride $\bar{d}_{x y}$ is fixed to $0.15 \mathrm{~m} .^{5}$ The desired torso attitude $\bar{\phi}$ is set to zero. The swinging leg is controlled by (14), and the yaw-moment is compensated by (21).

\footnotetext{
${ }^{5}$ We can make the switching stride vary, e.g. acc. the walking speed.
} 


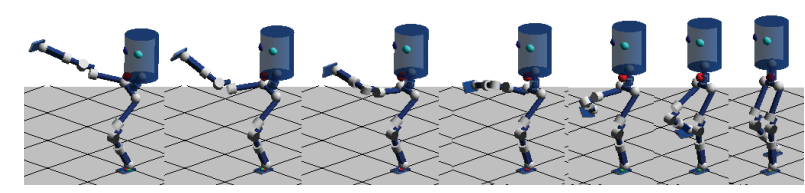

Fig. 9. Animation of a periodic reaching task by a leg with balance
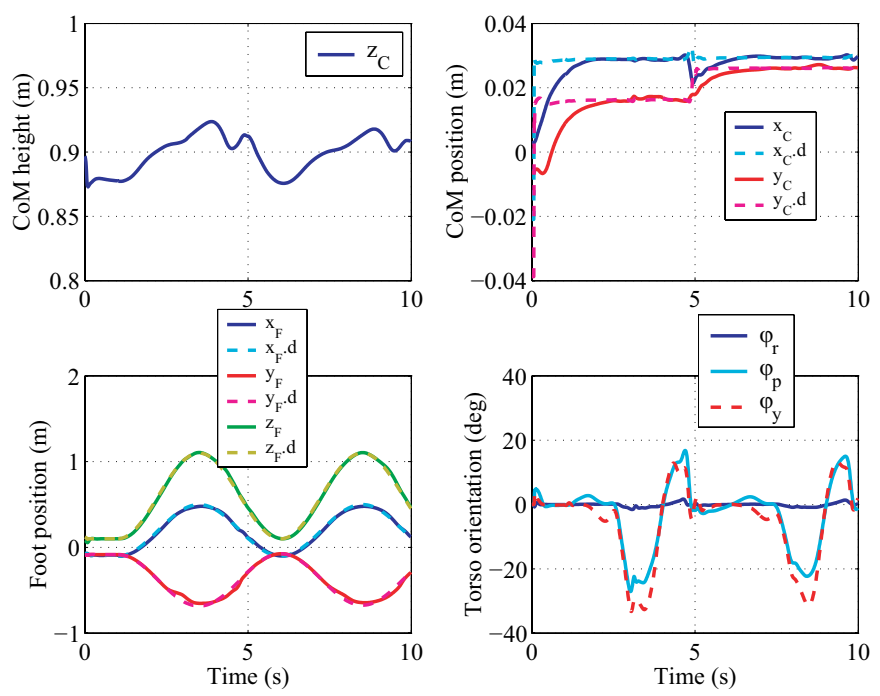

Fig. 10. Simulation result of a reaching task by a leg: the target foot positions indicated by $x_{F} . d, y_{F} . d, z_{F} . d$ are given by $0.2 \mathrm{~Hz}$ sinusoidal functions. The horizontal position of CoM $x_{C}, y_{C}$ is regulated to the center of the foot.

The robot does not fall, but walks to the direction of the external force. This is an externally-driven-walking, demonstrating the passivity of the closed-loop system. Note that the obtained walking gaits (periodic orbits) are not asymptotically stable, but Lyapnov stable. That is, for every neighborhood of walking gait, there exist family of other gaits as proven in [15]. The disturbances changes the heading of the robot (Fig. 5) as well as its acceleration, but the orientation of the base is well controlled by the yaw-moment compensation (21) so that the sagittal plane (Fig. 5) is aligned to the XZ-plane. However, note that the self-collision between limbs and the joint angle limits are disregarded. They may require another treatment, and left for the future work.

Since the Lyapnov stability, in the interested phase space limited by the fixed stride, is ensured in the bottom layer, it is trivial to achieve asymptotic stability in the upper layer (recall Section II). We only have to give $f_{u}$ in (23) to achieve some desired walking speed or reaching point. ${ }^{6}$ Fig. 12 shows such a simulation result where we set $\dot{\bar{x}}_{C}=1 \mathrm{~m} / \mathrm{s}$ in (23). A large amount of disturbance is applied. Nevertheless, the robot recovers the speed tracking after several steps.

We refer to [24] for its excellent performance of walking under a large disturbance. The advantages of our method over [24] are: 1) full-body force interaction without specifying

\footnotetext{
${ }^{6}$ In [15] a simple passivity-based controller via torso inclination was proposed.
}

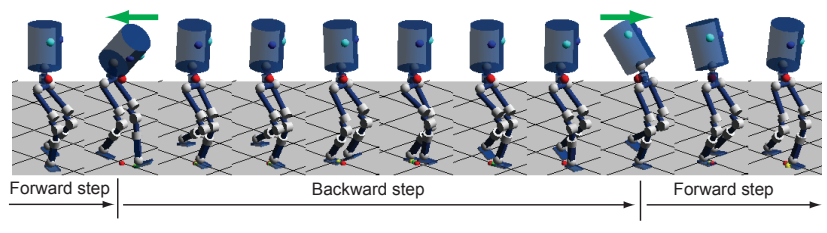

(a) Pushing from forward and backward

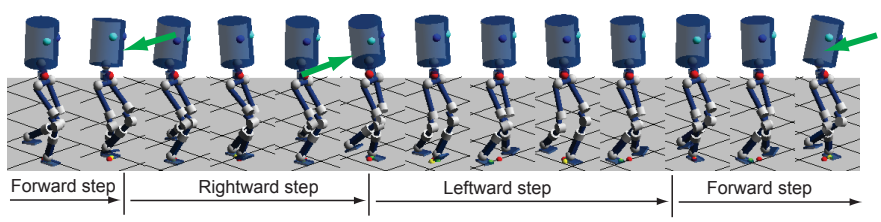

(b) Pushing from left, right and left
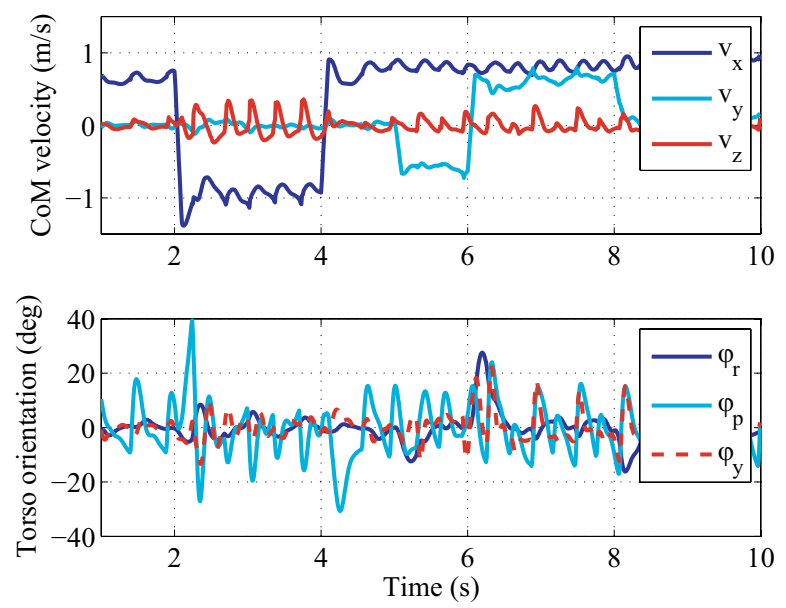

(c) The time history of the CoM velocities and the torso orientations

Fig. 11. The 3D symmetric walking simulation demonstrating its passivity against external force: During steady walking of $0.5 \mathrm{~m} / \mathrm{s}$, the robot is suddenly pushed from front by $1500 \mathrm{~N}$, then pushed $1500 \mathrm{~N}$ from back, $1000 \mathrm{~N}$ from left, and $1000 \mathrm{~N}$ from right and $500 \mathrm{~N}$ from left successively. The forces are applied to the base link (the application time is $0.1 \mathrm{~s}$ ).

some compensating joints in advance, 2) No joint trajectory calculation required (no pre-planned patterns at all), 3) The robot compliantly follows to the external force, then recovers as necessary, 4) No need to measure the external forces applied. We have also achieved even $2 \mathrm{~m} / \mathrm{s}$ walking (running) and continuous hopping in the simulation. Simulation movies are available from http://www.cns.atr.jp/ sangho/.

\section{CONCLUSION}

This paper proposed a passivity-based hierarchical fullbody motion controller for force-controllable humanoid robots. From a practical point of view, we have actually achieved:

- New force control framework allowed us to describe the 

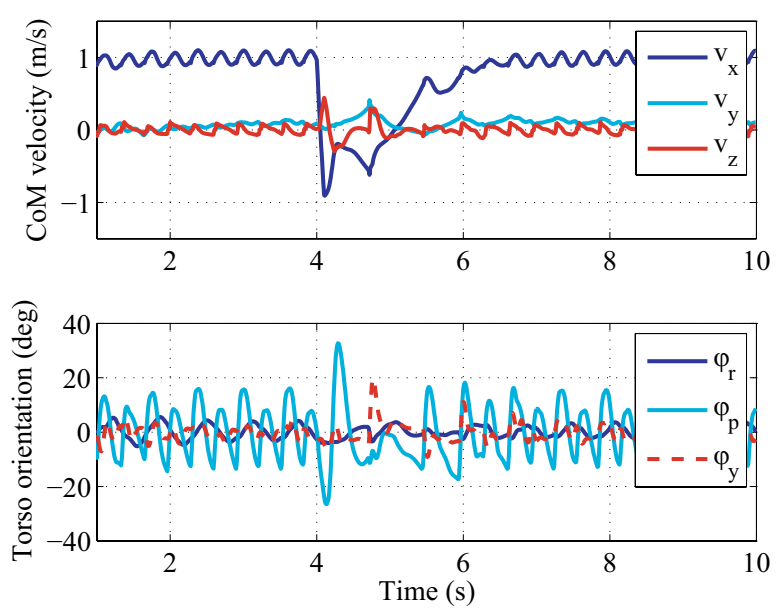

Fig. 12. The 3D symmetric walking with speed control. Even a large disturbance is applied $(-1500 \mathrm{~N}$ during $4-4.1 \mathrm{~s})$, the speed is converging to the desired value $(1 \mathrm{~m} / \mathrm{s})$.

contact force/moment, position, and orientation for either single or double support phase in a uniform manner;

- Different from position-based full-body controllers, the joint torque is automatically determied by the structure of Jacobian, without weighting coefficients;

- Passivity-based control strategy made it easy to prioritize and combine the each controllers for G-comp, balancing, orientation, and walking;

- SWC was successfully extended to multi-DOF humanoids and demonstrated the power of passivity and easy stabilization.

Four realistic dynamic simulations: balanced squatting, reaching, externally-driven and speed-controlled walking demonstrated the effectiveness of the proposed methods.

The force control framework proposed also works well in gymnastic motions and other dynamic force interaction with the environment since (12) combined with (13) can effectively compensate dynamic effect in the contact force control [16]. Further theoretical topics to be tackled are related to natural gait synthesis, which involves the difficult problems of: extension to ZMP-free balancer, joint torque minimization (natural contact states), joint limitation as well as self-collision avoidance, etc. The experimental results will be presented in the very near future [16].

The authors would like to thank the support of the Keihanna branch of the National Institute of Communication Telecommunication (NiCT), Japan.

\section{REFERENCES}

[1] M. Vukobratović and B. Borovac, "Zero-moment point - thirty five years of its life," International Journal of Humanoid Robotics, vol. 1, no. 1, pp. 157-173, 2004

[2] J. Yamaguchi, E. Soga, S. Inoue, and A. Takanishi, "Development of a bipedal humanoid robot-control method of whole body cooperative dynamic biped walking," in Proc. of IEEE International Conference on Robotics and Automation, May 1999, pp. 368 - 374.
[3] K. Hirai, M. Hirose, Y. Haikawa, and T. Takenaka, "The development of the honda humanoid robot," in Proc. of IEEE International Conference on Robotics and Automation, Leuven, Belgium, May 1998, pp. 13211328.

[4] H. Inoue, S. Tachi, Y. Nakamura, K. Hirai, N. Ohyu, Hirai.S., K. Tanie, K. Yokoi, and H. Hirukawa, "Overview of humanoid robotics project of METI," in The 32nd International Symposium on Robotics, 2001, pp. $1478-1482$.

[5] K. Mitobe, G. Capi, and Y. Nasu, "Control of walking robots based on manipulation of zero moment point," Robotica, vol. 18, no. 6, pp. 651-657, 2000

[6] K. Nagasaka, H. Inoue, and M. Inaba, "Dynamic walking pattern generation for a humanoid robot based on optimal gradient method," in Proc. of IEEE International Conference on Systems, Man, and Cybernetics, vol. 6, 12-15 Oct. 1999, pp. 908 - 913.

[7] T. Sugihara and Y. Nakamura, "Whole-body cooperative balancing of humanoid robot using COG Jacobian," in Proc. of IEEE/RSJ International Conference on Intelligent Robots and System, vol. 3, Lausanne, Swiss, Sept. 2002, pp. $2575-2580$.

[8] S. Kajita, F. Kanehiro, K. Kaneko, K. Fujiwara, K. Harada, K. Yokoi, and H. Hirukawa, "Resolved momentum control: humanoid motion planning based on the linear and angular momentum," in Proc. of IEEE/RSJ International Conference on Intelligent Robots and Systems, Las Vegas, USA, 2003, pp. 1644-1650.

[9] T. Ikeda, T. Tamura, and T. Mita, "Development and running control of a 3d leg robot,", in International Symposium on Adaptive Motion of Animals and Machines, Montreal, Canada, Aug. 2000, pp. FrP-2-3.

[10] S. Hyon, N. Yokoyama, and T. Emura, "Back handspring of a multilink gymnastic robot - reference model approach," Advanced Robotics, vol. 20, no. 1, pp. pp.93-113, 2006.

[11] L. Sentis and O. Khatib, "A whole-body control framework for humanoids operating in human environments -," in Proc. of IEEE International Conference on Robotics and Automation, Orlando, USA, May 2006, pp. 2641-2648

[12] G. Cheng, S. Hyon, J. Morimoto, A. Ude, and S. C. Jacobsen, "CB: A humanoid research platform for exploring neuroscience," in Proc. of IEEE-RAS International Conference on Humanoid Robots, Genova, Itary, 2006 (submitted).

[13] J. Morimoto, G. Endo, J. Nakanishi, S. Hyon, G. Cheng, D. Bentivegna, and C. G. Atkeson, "Modulation of simple sinusoidal patterns by a coupled oscillator model for biped walking," in Proc. of IEEE International Conference on Robotics and Automation, 2006 (Accepted).

[14] S. Hyon, "Hamiltonian-based running control of dynamic legged robots," Systems, Control and Information, vol. 49, no. 7, pp. 260-265, 2005 (in Japanese)

[15] S. Hyon and T. Emura, "Symmetric walking control: Invariance and global stability," in Proc. of IEEE International Conference on Robotics and Automation, Barcelona, Spain, Apr 2005, pp. 1455-1462.

[16] S. Hyon and G. Cheng, "Gravity compensation and whole-body force interaction for humanoid robots," in Proc. of IEEE-RAS International Conference on Humanoid Robots, Genova, Itary, 2006 (submitted).

[17] A. J. van der Schaft, L2-Gain and Passivity Techniques in Nonlinear Control. Springer, 1999

[18] S. Hyon and K. Fujimoto, "Hamiltonian locomotion," in SICE 6th Annual Conference on Control Systems, Nagoya, Japan, May 2006.

[19] J. Park and O. Khatib, "A whole-body control framework for humanoids operating in human environments," in Proc. of IEEE International Conference on Robotics and Automation, Orlando, USA, 2006, pp. 1963-1969.

[20] Greenwood, Classical Dynamics. Dover, 1977.

[21] R. M. Murray, Z. Li, and S. S. Sastry, A Mathematical Introduction to Robotic Manipulation. CRC Press, 1994.

[22] S. Arimoto, M. Sekimoto, H. Hashiguchi, and R. Ozawa, "Natural resolution of ill-posedness of inverse kinematics for redundant robots: a challenge to bernstein's degrees-of-freedom problem," Advanced Robotics, vol. 19 , no. 4 , pp. 401-434, 2005.

[23] J. G. Hale, "Contact handling with exact coulomb friction using constraints," in Proc. of IEEE-RAS International Conference on Humanoid Robots, Genova, Itary, 2006 (submitted).

[24] A. Takanishi, T. Takeya, H. Karaki, and I. Kato, "A control method for dynamic biped walking under unknown external force," in Proc. of IEEE International Workshop on Intelligent Robots and Systems, July 1990, pp. $795-801$. 